ISSN 0103-5150

Fisioter. Mov., Curitiba, v. 24, n. 3, p. 523-533, jul./set. 2011 Licenciado sob uma Licença Creative Commons

\title{
PEDro: a base de dados de evidências em fisioterapia
}

\author{
PEDro: the physiotherapy evidence database
}

\section{Sílvia Regina Shiwa ${ }^{[a]}$, Leonardo Oliveira Pena Costa ${ }^{[b]}$, Auristela Duarte de Lima Moser ${ }^{[c]}$, Isabella de Carvalho Aguiar $^{[\mathrm{d}]}$, Luis Vicente Franco de Oliveira ${ }^{[\mathrm{e}]}$}

[a] Mestranda em Fisioterapia, Universidade Cidade de São Paulo (UNICID), São Paulo, SP - Brasil, e-mail: silvia.shiwa@gmail.com

[b] Coordenador do Programa de Mestrado em Fisioterapia, Centro Brasileiro de Fisioterapia Baseada em Evidências/PEDro Brasil, Universidade Cidade de São Paulo (UNICID), São Paulo, SP - Brasil.

[c] Professora do Programa de Pós Graduação em Tecnologia em Saúde (PUCPR), Curitiba, PR - Brasil.

[d] Mestranda em Ciências da Reabilitação, Universidade Nove de Julho (UNINOVE), São Paulo, SP - Brasil.

[e] Professor do Programa de Mestrado e Doutorado em Ciências da Reabilitação (UNINOVE), São Paulo, SP, Brasil.

\section{Resumo}

Introdução: A prática baseada em evidências é necessária para que os pacientes recebam tratamentos eficazes, assim como para reduzir os custos de saúde em geral. Com o crescimento da produção científica com qualidade metodológica heterogênea, torna-se difícil para o fisioterapeuta selecionar quais as melhores evidências para embasar a sua conduta terapêutica. Objetivos: Apresentar a base de dados PEDro (Physiotherapy Evidence Database). Materiais e métodos: Foram descritos a abrangência da base de dados, tipos de estudos indexados, grau de acessibilidade aos usuários e critérios de qualificação metodológica, entre outras características. Resultados: PEDro é a base de dados mais abrangente em estudos que testam a eficácia das intervenções fisioterapêuticas, em que são indexados estudos controlados aleatorizados, revisões sistemáticas e diretrizes de prática clínica, e os estudos controlados aleatorizados possuem sua qualidade metodológica e descrição estatística avaliadas por meio da escala de qualidade PEDro. A PEDro tem acesso livre e gratuito, facilitando o uso por profissionais, alunos da área e pesquisadores. Os resultados das buscas são ranqueados de acordo com a qualidade metodológica dos artigos encontrados. A PEDro está disponível em cinco idiomas: inglês, mandarim, francês, alemão e português e disponibiliza uma versão simples, sem termos técnicos, para consumidores de serviços de fisioterapia, que são as "escolhas 
fisioterapêuticas", ou physiotherapy choices. Conclusão: A PEDro cumpre o seu papel auxiliando tanto profissionais como pacientes, informando sobre as evidências de eficácia de tratamentos fisioterapêuticos de uma forma rápida e simples e contribuindo com o recolhimento de informações para subsidiar decisões ou escolhas terapêuticas.

Palavras-chave: Prática clínica baseada em evidências. Base de dados. Fisioterapia.

\section{Abstract}

Introduction: Evidence-based practice is needed in order to provide effective and low cost treatments for patients. Due to the growth of the number scientific articles with heterogeneous methodological quality, it becomes difficult for physiotherapists to select which information to be used in order to keep updated as well as to make clinical decisions. Objective: The aim of this article is to present the Physiotherapy Evidence Database (PEDro). Method: We described the comprehensiveness, types of studies indexed, the accessibility and how the methodological quality of randomised controlled trials is rated on the PEDro database. Results: PEDro is the most comprehensive database that indexes studies aimed to test the efficacy of physiotherapy interventions. PEDro indexes randomized controlled trials, systematic reviews and clinical practice guidelines, being the randomized controlled trials having their methodological quality and statistical reporting evaluated by the PEDro scale. PEDro is an open access database, which facilitates the use for physiotherapists, students and researchers. The search results are ranked according to the methodological quality. The PEDro website is available in English, Mandarin, French, German and Portuguese and the website also have an easy-friendly using link to the consumer version of PEDro, named "physiotherapy choices". Conclusion: PEDro database helps both health care professionals as well as patients by informing them with regards to the efficacy of physiotherapy interventions in an easy and quick way. PEDro contributes significantly in the decision-making process by providing all information available regarding the efficacy of physiotherapy interventions.

Keywords: Evidence-based practice. Database. Physiotherapy.

\section{Introdução}

A fisioterapia baseada em evidências é o elemento central da fisioterapia contemporânea. A aplicação de intervenções baseadas em evidências científicas é necessária para que os pacientes recebam tratamentos eficazes, assim como para reduzir os custos de saúde em geral. As revisões sistemáticas, as diretrizes de prática clínica e os estudos controlados aleatorizados (ECAs) (Quadro 1) são considerados as melhores fontes de evidência para avaliar o efeito de uma determinada intervenção (1), isto é, se os tratamentos são eficazes ou não. Sugere-se que esses estudos devem sempre ser escolhidos para auxiliar fisioterapeutas em suas tomadas de decisão clínica (2).

0 primeiro ECA em fisioterapia foi publicado em 1929, por Colebrook e colaboradores, no periódico Medical Research Council Special Report Series. Esse estudo investigou o efeito da irradiação ultravioleta na prevenção de resfriados, doenças infecciosas, der- matofitoses e promoção do progresso escolar (3). Desde então, o número de ECAs com o objetivo de testar intervenções fisioterapêuticas cresceu rapidamente. Em 1960, havia 15 ECAs e, nas quatro décadas seguintes, esse número teve um crescimento exponencial, com 86 ECAs em 1970, 441 em 1980, 1.925 em 1990 e 5.301 em 2000. As revisões sistemáticas de ECAs relevantes para a fisioterapia também acompanharam esse crescimento. A primeira revisão sistemática foi publicada em 1975 (4), e em 1991 havia 39 revisões. A primeira diretriz de prática clínica foi publicada em 1987 (5). Em 1995 e 2009 totalizavam 19 e 603 diretrizes de prática clínica, respectivamente (6). Em maio de 2011 havia 2.801 revisões sistemáticas, 808 diretrizes de prática clínica e 15.293 ECAs relacionados a intervenções fisioterapêuticas.

Com base nesses dados é possível observar um aumento considerável no número de informações sobre a eficácia de intervenções em fisioterapia e não há previsão para redução desse crescimento em um 
futuro próximo (Gráfico 1), uma vez que o número de estudos relacionados à eficácia de intervenções dobra a cada três anos e meio. Diante dessa situação tão dinâmica, é improvável que fisioterapeutas consigam lidar com esse expressivo volume de informações. Além do grande número de estudos relacionados à eficácia de intervenções, outro problema encontrado por fisioterapeutas que desejam se atualizar adequadamente é o fato de que a qualidade metodológica dos estudos tende a ser muito heterogênea, sendo a maioria dos estudos de baixa qualidade metodológica. Esses estudos de baixa qualidade metodológica proporcionam conclusões com pouca validade científica, que infelizmente mais confundem do que orientam tomadas de decisão clínica de fisioterapeutas. É importante salientar que esses problemas são também observados em outras profissões da área da saúde.

Uma solução que pode amenizar esses problemas seria o ranqueamento quanto à qualidade metodológica dos estudos relacionados à eficácia de intervenções em fisioterapia, e que esses resultados estejam disponíveis em tempo real e sem custo para toda a comunidade de fisioterapeutas. Felizmente, essa solução já é disponível através da base de dados PEDro (Physiotherapy Evidence Database).

Cientes dessa necessidade, os autores deste estudo definiram como objetivo: uma exploração prática da base de dados PEDro, descrever os tipos de estudos indexados, grau de acessibilidade aos usuários e critérios de qualificação metodológica com vistas à sua apresentação aos leitores deste periódico.

Os autores realizaram o acesso à base tendo em mente simular uma busca para apresentar o funcionamento da base de dados, assim como apresentar os tipos de estudos indexados, grau de acessibilidade aos usuários e critérios de qualificação metodológica. Os resultados são apresentados a seguir.

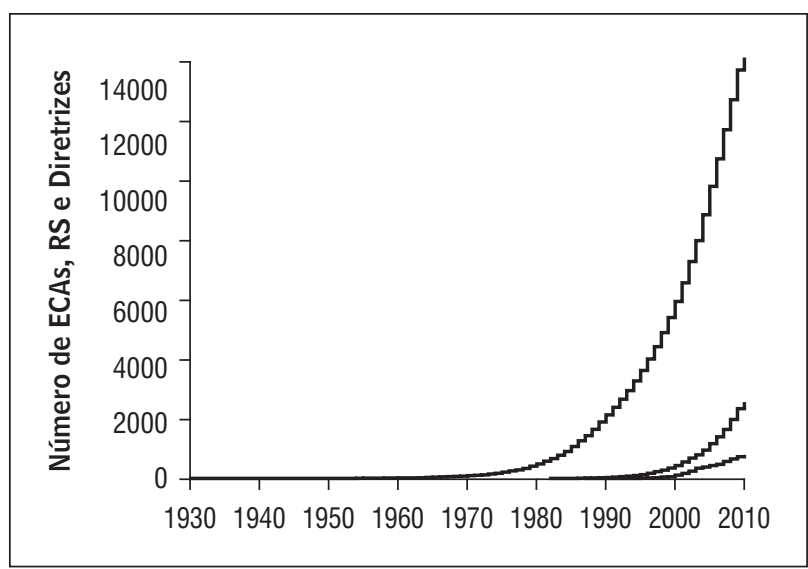

Gráfico 1 - Frequência acumulada do número de estudos controlados aleatorizados (ECAs), revisões sistemáticas (RS) e diretrizes de prática clínica em fisioterapia por década

Quadro 1 - Definição dos tipos de estudos relevantes para avaliar a eficácia de intervenções

\begin{tabular}{ll}
\hline Tipo de estudo & Definição \\
\hline $\begin{array}{l}\text { Estudo controlado } \\
\text { aleatorizado (ECA) }\end{array}$ & $\begin{array}{l}\text { São estudos longitudinais, de intervenção, controlados, em que os pacientes são distribuídos em dois ou } \\
\text { mais grupos de forma aleatória (os grupos controle podem ser um grupo que não recebe tratamento, que } \\
\text { recebe um tratamento placebo ou que recebe outro tipo de tratamento). Os ECAs são considerados como } \\
\text { padrão ouro dos estudos que têm como objetivo avaliar efeitos de intervenções terapêuticas. }\end{array}$ \\
\hline Revisão sistemática & $\begin{array}{l}\text { É um modo de executar revisões abrangentes da literatura de forma não tendenciosa. Reúne de forma orga- } \\
\text { nizada uma grande quantidade de resultados de ECAs. Uma revisão sistemática responde a uma pergunta } \\
\text { claramente formulada, utilizando métodos sistemáticos e explícitos para identificar, selecionar e avaliar } \\
\text { criticamente pesquisas relevantes, assim como coletar e analisar dados de estudos incluídos na revisão. } \\
\text { Os métodos estatísticos (meta-análise) podem ou não ser usados para analisar e sumarizar os resultados } \\
\text { dos estudos incluídos. }\end{array}$ \\
\hline $\begin{array}{l}\text { Diretriz de prática clínica } \\
\text { (Guidelines) }\end{array}$ & $\begin{array}{l}\text { É um conjunto de recomendações para a prática clínica (prevenção, diagnóstico e tratamento). Cada uma } \\
\text { das recomendações deve estar embasada pela melhor evidência científica disponível, representando orien- } \\
\text { tações para a prática baseada em evidências. As diretrizes devem tornar mais fáceis as decisões na prática } \\
\text { diária, devem ser concretas, precisas, aplicáveis em todo o território nacional, devem alertar para os riscos, } \\
\text { limitações, possíveis prejuízos e benefícios de sua aplicação. Podem ser consideradas como um caminho } \\
\text { que auxilia o profissional a separar as práticas desnecessárias das necessárias. }\end{array}$ \\
\hline
\end{tabular}

Fonte: Ministério da Educação (19). 


\section{Resultados}

\section{O que é a PEDro?}

A PEDro é uma base de dados específica para estudos que investigam a eficácia de intervenções em fisioterapia, podendo ser acessada gratuitamente por meio do site <http://www.pedro.org.au>. Essa base de dados foi criada em 1999, por um grupo de fisioterapeutas australianos do Centro de Fisioterapia Baseada em Evidências da Universidade de Sydney, com a missão de maximizar a eficácia dos serviços de fisioterapia e facilitar a aplicação prática da melhor evidência existente, com o lema principal "a fisioterapia eficaz deve ser centrada no paciente, focado na prevenção, baseada na melhor evidência existente e gerenciada de forma eficiente" (7).

Dois estudos apontam a PEDro como a base de dados mais abrangente de estudos que testam a eficácia das intervenções fisioterapêuticas $(8,9)$. Esses estudos compararam a abrangência de diversas bases de dados, entre elas, AMED, CENTRAL, CINAHL, PubMed, Hooked on Evidence, Embase, PsycINFO e a PEDro. Moseley e colaboradores (9) concluíram que a PEDro e a CENTRAL (a base de dados de ECAs da colaboração Cochrane) são as bases de dados mais completas quanto à indexação de ECAs de fisioterapia. Em outro estudo, Michaleff e colaboradores (8) concluíram que as bases CENTRAL, PEDro, PubMed e Embase são as mais abrangentes para realizar as buscas de ECAs de intervenções fisioterapêuticas, destacando que, dessas quatro bases, a PEDro é a única específica de estudos relacionados à fisioterapia.

A PEDro é utilizada por fisioterapeutas em mais de 80 países, com mais de 4.300 buscas realizadas por dia pelo website, tendo fornecido no último ano respostas para mais de 800.000 perguntas clínicas (10). Dentre esses países, destacam-se a Austrália, os Estados Unidos e o Brasil como os três que mais acessam a base, respectivamente, sendo aproximadamente $10 \%$ de todos os acessos da base de dados PEDro realizados por fisioterapeutas brasileiros.

Na PEDro estão indexados cerca de 19.000 estudos controlados aleatorizados, revisões sistemáticas e diretrizes da prática clínica em fisioterapia. Quando possível, são colocados a disposição dos usuários o resumo e o link para o texto completo de cada artigo. A razão disso é que nem sempre a PEDro recebe autorização dos editores dos periódicos cientí- ficos para publicar o resumo ou mesmo o link para o texto completo. Todos os estudos controlados aleatorizados possuem sua qualidade metodológica e descrição estatística avaliados por meio da escala de qualidade PEDro (Quadro 2). Ao realizar uma busca, esses estudos são ranqueados em ordem de importância metodológica para facilitar o acesso rápido à evidência mais válida possível.

Para um ECA ser inserido na base de dados PEDro, ele deve obedecer a cinco critérios:

1) o estudo deve comparar no mínimo duas intervenções terapêuticas;

2) pelo menos uma das intervenções que estão sendo testadas pelo estudo deve fazer parte do arsenal terapêutico dos fisioterapeutas;

3) as intervenções dos estudos devem ser aplicadas em seres humanos que representem a população de pacientes que frequentemente utilizam serviços de fisioterapia;

Quadro 2 - Escala de qualidade PEDro (versão em português)

\section{Escala de qualidade PEDro - português (Brasil)}

1. Os critérios de elegibilidade foram especificados.

2. Os sujeitos foram aleatoriamente distribuídos por grupos (em um estudo cruzado, os sujeitos foram colocados em grupos, de forma aleatória, de acordo com o tratamento recebido).

3. A alocação dos sujeitos foi secreta.

4. Inicialmente, os grupos eram semelhantes no que diz respeito aos indicadores de prognóstico mais importantes.

5. Todos os sujeitos participaram de forma cega no estudo.

6. Todos os terapeutas que administraram a terapia fizeram-no de forma cega.

7. Todos os avaliadores que mediram pelo menos um resultado-chave fizeram-no de forma cega.

8. Mensurações de pelo menos um resultado-chave foram obtidas em mais de $85 \%$ dos sujeitos inicialmente distribuídos pelos grupos.

9. Todos os sujeitos a partir dos quais se apresentaram mensurações de resultados receberam o tratamento ou a condição de controle conforme a alocação ou, quando não foi esse 0 caso, fez-se a análise dos dados para pelo menos um dos resultados-chave por "intenção de tratamento".

10. Os resultados das comparações estatísticas intergrupos foram descritos para pelo menos um resultado-chave.

11. 0 estudo apresenta tanto medidas de precisão como medidas de variabilidade para pelo menos um resultado-chave. 
4) a distribuição dos sujeitos nos grupos de tratamento e controle deve ser realizada de forma aleatória ou com "intenção de ser aleatória";

5) o estudo deve estar publicado em formato integral em revista revisada por pares.1 Após a inclusão do estudo na base de dados PEDro, este é avaliado quanto à sua qualidade metodológica e descrição estatística por meio da escala de qualidade PEDro.

Para inserção de revisões sistemáticas, deve-se obedecer a dois critérios:

1) ser uma revisão sistemática de ECAs; e

2) ter uma sessão de métodos e que pelo menos um artigo da revisão satisfaça aos cinco critérios de inclusão de estudos controlados aleatorizados da base de dados PEDro (6).

Para a inserção das diretrizes de prática clínica, deve-se obedecer a seis critérios:

1) ter sido produzido sob o controle de uma associação especializada da área, sociedade profissional, organização pública ou privada, órgãos de governo, ou instituições de saúde em geral. Se a diretriz de prática clínica foi desenvolvida por um indivíduo ou grupo de indivíduos não oficialmente apoiados por um dos tipos de organizações citadas anteriormente, essa não será incluída na PEDro;
2) estar disponível publicamente;

3) a busca sistemática da literatura deve ser baseada em artigos publicados em revistas revisadas por pares ou ser baseada em uma revisão sistemática publicada nos quatro anos anteriores à publicação da diretriz;

4) pelo menos um ECA relacionado com a fisioterapia deve estar incluído na diretriz;

5) a diretriz deve conter afirmações sistematicamente desenvolvidas, que incluem recomendações, estratégias e informações que auxiliem os fisioterapeutas ou pacientes a tomar decisões;

6) pelo menos uma recomendação deve ser feita para uma intervenção que faz parte do arsenal da fisioterapia ou que poderia se tornar parte da prática fisioterapêutica.

\section{Escala de qualidade PEDro}

A grande parte dos critérios da escala de qualidade PEDro baseia-se na escala Delphi, desenvolvida por Verhagen e colaboradores (11), do Departamento de Epidemiologia da Universidade de Maastricht, Holanda, com exceção de dois critérios. A escala baseia-se em um "consenso de especialistas" e não em dados empíricos (Quadro 3).

A escala de qualidade PEDro inclui dois critérios adicionais que não constavam da escala Delphi: um critério relativo ao número de pacientes avaliados em cada período de acompanhamento (critério 8)

Quadro 3 - Indicações para a administração da escala PEDro

\begin{tabular}{ll} 
Todos os critérios & $\begin{array}{l}\text { A pontuação só será atribuída quando um critério for claramente satisfeito. Se em uma leitura literal do relatório do } \\
\text { ensaio existir a possibilidade de um critério não ter sido satisfeito, ele não deve receber pontuação. }\end{array}$ \\
\hline Critério 1 & $\begin{array}{l}\text { Esse critério pode considerar-se satisfeito quando o relatório descreve a origem dos sujeitos e a lista de requisitos } \\
\text { utilizados para determinar quais sujeitos eram elegíveis para participar do estudo. }\end{array}$ \\
\hline Critério 2 & $\begin{array}{l}\text { Considera-se que em um determinado estudo houve alocação aleatória se o relatório referir que a alocação dos } \\
\text { sujeitos foi aleatória. } 0 \text { método de aleatoriedade não precisa ser explícito. Procedimentos tais como lançamento } \\
\text { de dados ou moeda ao ar podem ser considerados como alocação aleatória. Procedimentos de alocação quase- } \\
\text { aleatória, tais como os que se efetuam a partir do número de registro hospitalar, da data de nascimento, ou de } \\
\text { alternância, não satisfazem esse critério. }\end{array}$ \\
\hline Critério 3 & $\begin{array}{l}\text { Alocação secreta significa que a pessoa que determinou a elegibilidade do sujeito para participar no ensaio des- } \\
\text { conhecia, quando a decisão foi tomada, o grupo a que o sujeito iria pertencer. Deve atribuir-se um ponto a esse } \\
\text { critério, mesmo que não se diga que a alocação foi secreta, quando o relatório refere que a alocação foi feita a partir } \\
\text { de envelopes opacos fechados ou que implicou o contato com o responsável pela alocação dos sujeitos por grupos } \\
\text { e esse último não participou do ensaio. }\end{array}$
\end{tabular}


Critério 4

No mínimo, nos estudos de intervenções terapêuticas, o relatório deve descrever pelo menos uma medida da gravidade da condição a ser tratada e pelo menos uma (diferente) medida de resultado-chave que caracterize a linha de base. 0 examinador deve assegurar-se de que, com base nas condições de prognóstico de início, não seja possível prever diferenças clinicamente significativas dos resultados para os diversos grupos. Esse critério é atingido mesmo que somente sejam apresentados os dados iniciais do estudo.

Critérios 4, 7-11 Resultados-chave são resultados que fornecem o indicador primário da eficácia (ou falta de eficácia) da terapia. Na maioria dos estudos, utilizam mais do que uma variável como medida de resultados.

Critérios 5-7 Ser cego para o estudo significa que a pessoa em questão (sujeito, terapeuta ou avaliador) não sabe qual o grupo a que 0 sujeito pertence. Mais ainda, sujeitos e terapeutas só são considerados "cegos" se for possível esperar que sejam incapazes de distinguir entre os tratamentos aplicados aos diferentes grupos. Nos ensaios em que os resultados-chave são relatados pelo próprio (por exemplo, escala visual análoga, registro diário da dor), 0 avaliador é considerado "cego" se o sujeito foi "cego".

Critério 8

Esse critério só se considera satisfeito se o relatório referir explicitamente tanto o número de sujeitos inicialmente alocados nos grupos como o número de sujeitos a partir dos quais se obtiveram medidas de resultados-chave. Nos ensaios em que os resultados são medidos em diferentes momentos no tempo, um resultado-chave tem de ter sido medido em mais de $85 \%$ dos sujeitos em algum desses momentos.

Critério 9

Uma análise de intenção de tratamento significa que, quando os sujeitos não receberam tratamento (ou a condição de controle) conforme o grupo atribuído e quando se encontram disponíveis medidas de resultados, a análise foi efetuada como se os sujeitos tivessem recebido o tratamento (ou a condição de controle) que lhes foi atribuído inicialmente. Esse critério é satisfeito, mesmo que não seja referida a análise por intenção de tratamento, se o relatório referir explicitamente que todos os sujeitos receberam o tratamento ou condição de controle, conforme a alocação por grupos.

Critério 10

Uma comparação estatística intergrupos implica uma comparação estatística de um grupo com outro. Conforme o desenho do estudo, isso pode implicar uma comparação de dois ou mais tratamentos ou a comparação do tratamento com a condição de controle. A análise pode ser uma simples comparação dos resultados medidos após a administração do tratamento ou a comparação das alterações em um grupo em relação às alterações no outro (quando se usa uma análise de variância para analisar os dados, essa última é frequentemente descrita como interação grupo versus tempo). A comparação pode apresentar-se sob a forma de hipóteses (por meio de um valor de $p$, descrevendo a probabilidade dos grupos diferirem apenas por acaso) ou assumir a forma de uma estimativa (por exemplo, a diferença média ou a diferença mediana, ou uma diferença nas proporções, ou um número necessário para tratar, ou um risco relativo ou uma razão de risco) e respectivo intervalo de confiança.

Critério 11

Uma medida de precisão é uma medida da dimensão do efeito do tratamento. Já este pode ser descrito como uma diferença nos resultados do grupo ou como o resultado em todos os (ou em cada um dos) grupos. Medidas de variabilidade incluem desvios-padrão (DPs), erros-padrão (EPs), intervalos de confiança, amplitudes interquartis (ou outras amplitudes de quantis) e amplitudes de variação. As medidas de precisão e/ou as medidas de variabilidade podem ser apresentadas graficamente (por exemplo, os DPs podem ser apresentados como barras de erro em uma figura) desde que aquilo que é representado seja inequivocamente identificável (por exemplo, desde que fique claro se as barras de erro representam DPs ou EPs). Quando os resultados são relativos a variáveis categóricas, considera-se que esse critério foi cumprido se o número de sujeitos em cada categoria é apresentado para cada grupo.

e um outro critério que avalia se houve comparação estatística entre grupos (critério 10). Apesar de essa escala não avaliar a validade externa do estudo, o critério relativo à elegibilidade dos participantes (critério 1) foi mantido para que todos os critérios da escala Delphi estejam representados na escala de qualidade PEDro.

O objetivo da escala de qualidade PEDro é auxiliar os usuários da base de dados PEDro quanto à quali- dade metodológica dos ECAs (validade interna, critérios 2 a 9 da escala), bem como avaliar a descrição estatística, isto é, se o estudo contém informações estatísticas mínimas para que os resultados possam ser interpretáveis (critérios 10 e 11 da escala). Não são avaliadas a validade externa do estudo, generalização dos resultados, nem a magnitude do efeito de tratamento (isto é, se os resultados são clinicamente relevantes ou não). 
Os avaliadores da PEDro analisam somente o que está reportado no manuscrito, quando há dúvida por parte do avaliador na hora de pontuar o critério, ele o classifica como "não", obedecendo a recomendação de escala de qualidade metodológica denominada: "culpado até que se prove a inocência". Por exemplo, se um estudo reportar que é "duplo cego", mas não explicar quem foi cego, receberá a classificação de "não" para todos os critérios relacionados ao cegamento de pacientes, terapeutas e avaliadores. A pontuação final da escala de qualidade PEDro é dada por meio da soma do número de critérios que foram classificados como satisfatórios entre os critérios 2 ao 11 . 0 critério 1 não é considerado para a pontuação final por tratar-se de um item que avalia a validade externa do estudo. Cada ECA é avaliado por dois avaliadores independentes e, no caso de discordância de avaliação de qualquer item em questão, um terceiro avaliador faz uma arbitragem final. A pontuação final pode variar entre 0 e 10 pontos.

A escala de qualidade PEDro em inglês possui propriedades de medida satisfatórias, apresentando uma moderada confiabilidade entre examinadores. A validade da escala já foi testada e confirmada em dois estudos $(12,13)$. Esses dados confirmam que ela é capaz de distinguir entre ECAs de boa qualidade e de má qualidade. As propriedades clinimétricas da escala de qualidade PEDro em português estão sendo testadas pelo nosso grupo de pesquisa e os resultados serão divulgados em breve (14).

Essa mesma escala não deverá ser usada para a interpretação dos resultados de ECAs. Advertimos aos usuários dessa escala que estudos que revelem efeitos estatisticamente significativos do tratamento e que obtenham pontuação elevada na escala PEDro não fornecem necessariamente evidência de que o tratamento seja clinicamente útil. Adicionalmente, importa saber se o efeito do tratamento foi suficientemente eficaz para poder ser considerado clinicamente relevante, se os efeitos positivos superam os negativos, e aferir a relação de custo-benefício do tratamento.

Dois critérios da PEDro - o cegamento do terapeuta e do paciente - nem sempre são possíveis de serem cumpridos durante a realização dos estudos. Por exemplo, um estudo comparando duas intervenções para dor lombar, tendo um grupo de pacientes recebido exercícios e o outro grupo recebido uma cartilha de orientações. Nesse exemplo foi possível que pacientes e terapeutas conseguissem distinguir os dois tipos de tratamento oferecido. Portanto, a pontuação máxima na escala PEDro que esse estudo poderia receber seria de 8 pontos, pois os pontos relacionados ao cegamento de pacientes e terapeutas sequer podem ser cumpridos e automaticamente recebem "não". Outro exemplo seria um estudo comparando a eficácia de um equipamento de eletroterapia com placebo, comparação do uso TENS convencional versus TENS em dose mínima. Nesse caso é possível cegar o paciente e o terapeuta, uma vez que estes seriam incapazes de distinguir as duas intervenções, pois não reconheceriam qual equipamento é o convencional e qual é o placebo. Dessa forma, esse artigo pode receber a pontuação máxima na escala PEDro de 10 pontos. Sendo assim, a escala não deve ser utilizada para comparar a "qualidade" de ECAs realizados em diferentes áreas de terapia, principalmente porque em algumas áreas da prática da fisioterapia não é possível satisfazer todos os itens da escala.

\section{Indexações PEDro}

A base de dados PEDro indexa estudos em diversas línguas e os 19.026 estudos que estão na base atualmente foram publicados em 34 idiomas diferentes. Os idiomas mais comumente encontrados nos artigos da base são: inglês (90\%), mandarim (3\%), alemão $(2 \%)$, holandês $(1 \%)$, francês $(1 \%)$ e português $(0,6 \%)$. É importante ressaltar que a PEDro é uma base de dados que somente indexa ECAs, revisões sistemáticas e diretrizes de prática clínica. Qualquer outro tipo de delineamento, tais como estudos de caso-controle, estudos observacionais relacionados à fisiologia ou biomecânica, estudos de caso único, estudos de coorte, estudos com indivíduos normais, experimentos com animais, revisões narrativas, não são indexados na PEDro.

Apesar desses tipos de estudos serem extremamente relevantes para a fisioterapia, eles não são os melhores para estabelecer se uma determinada intervenção é eficaz ou não e, portanto, não fazem parte dos estudos que são indexados na base de dados PEDro $(2,15)$.

\section{Qualidade dos estudos controlados aleatorizados}

Apesar do grande volume de ECAs na área da fisioterapia, a maior parte desses trabalhos são de baixa qualidade, problema esse que se agrava quando 
analisadas as publicações nacionais. Um estudo do nosso grupo de pesquisa analisou 50 ECAs de fisioterapia publicadas em português por meio da escala de qualidade PEDro, a pontuação média dos estudos foi de 3,4 pontos (DP 1,4), de um total de 10 pontos possíveis. Também observamos que $84 \%$ desses estudos apresentaram nota 4 ou inferior (14). Os valores encontrados nessas publicações nacionais apresentam-se inferiores à média mundial, que é de 5,2 pontos (DP 1,6) (1). Pode-se concluir que ainda há uma necessidade de se melhorar a qualidade metodológica e as apresentações estatísticas dos ECAs nacionais e internacionais.

\section{Como fazer uma busca na PEDro?}

$\mathrm{Na}$ base de dados PEDro é possível realizar a busca de duas formas: busca simples ou busca avançada, estando atualmente as páginas do sistema de busca em inglês. Ao acessar o website da PEDro e clicar em "simple search" (busca simples - Figura 1), aparecerá uma página com uma caixa de texto para que sejam escritos os termos de busca sobre o tópico a ser pesquisado na PEDro, devendo ser digitados os termos de busca em inglês. Quando forem colocados um ou mais termos, a PEDro realizará uma busca dos artigos que contenham essas palavras, lembrando que, se for colocado mais de um termo, será buscado por artigos que contenham todas as palavras inseridas.

Depois, ao clicar em "start search" (iniciar a pesquisa), aparecerá uma lista com todos os artigos encontrados, ranqueados pela qualidade metodológica (primeiro as diretrizes, seguidas das revisões sistemáticas e depois os ECAs ordenados pela escala PEDro - Figura 2). Ao clicar sobre o título do artigo, aparecerão os dados bibliométricos do artigo, assim como os critérios da escala de qualidade PEDro que foram satisfatórios (Figura 3). Um exemplo pode ser visto nas Figuras 1 a 3, que apresentam o resultado de uma busca por evidências do uso do ultrassom no tratamento das epicondilites.

Para busca avançada é necessário clicar em "advanced search" (Figura 4). Aparecerá uma página com 12 campos de busca, podendo qualquer um ser utilizado para realizar a busca, não sendo necessário o preenchimento de todos os campos. Os campos

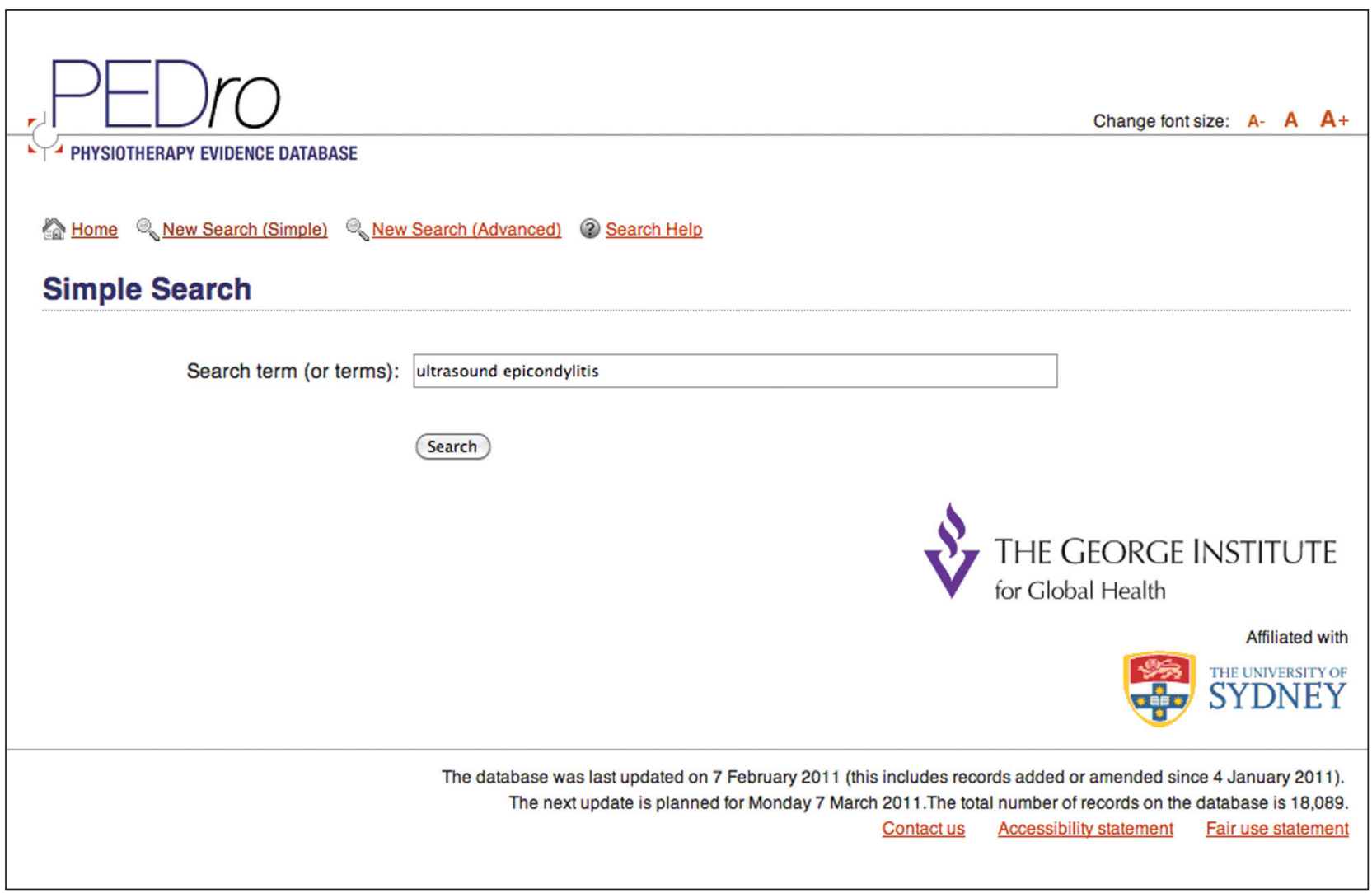

Figura 1 - Página do site PEDro: busca simples 


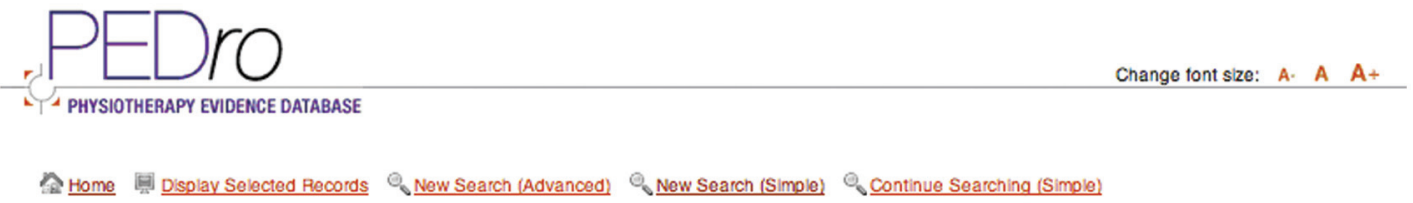

\section{Search Results}

Click on a title to view details of that record. If your search has returned many records you may need to click on Next (at the top or bottom of the list of records). To display a list of records from one or a series of searches, click on Select and then Display Selected Records (at the top of the page).

Record $1-23$ of 23

Title

Rehabilitation for patients with lateral epicondylitis: a systematic review

Effectiveness of physiotherapy for lateral epicondylitis: a systematic review [with consumer summany]

Deep transverse friction massage for treating tendinitis (Cochrane review) [with consumer summany]

Ultrasound therapy for musculoskeletal disorders: a systematic review

Randomized controlled trial of a new electrical modality (InterX) and soft tissue massage, stretching ultrasound and exercise for treating lateral epicondylitis

Pulsed low-intensity ultrasound therapy for chronic lateral epicondylitis: a randomized controlled trial

Manipulation of the wrist for management of lateral epicondylitis: a randomized pilot study

$\begin{array}{lll}\begin{array}{lll}\text { Method } & \underline{\text { Score }} & \text { Select } \\ \text { Record }\end{array} \\ \begin{array}{l}\text { systematic N/A } \\ \text { review }\end{array} & \underline{\text { Select }} \\ \begin{array}{l}\text { systematic N/A } \\ \text { review }\end{array} & \underline{\text { Select }} \\ \begin{array}{l}\text { systematic N/A } \\ \text { review }\end{array} & \text { Select } \\ \begin{array}{l}\text { systematic N/A } \\ \text { review }\end{array} & \text { Select } \\ \begin{array}{l}\text { clinical } \\ \text { trial }\end{array} & \\ \begin{array}{l}\text { clinical } \\ \text { trial }\end{array} & \text { 7/10 } & \text { Select } \\ \begin{array}{l}\text { clinical } \\ \text { trial }\end{array} & 7 / 10 & \text { Select } \\ \end{array}$

Figura 2 - Apresentação do resultado da busca

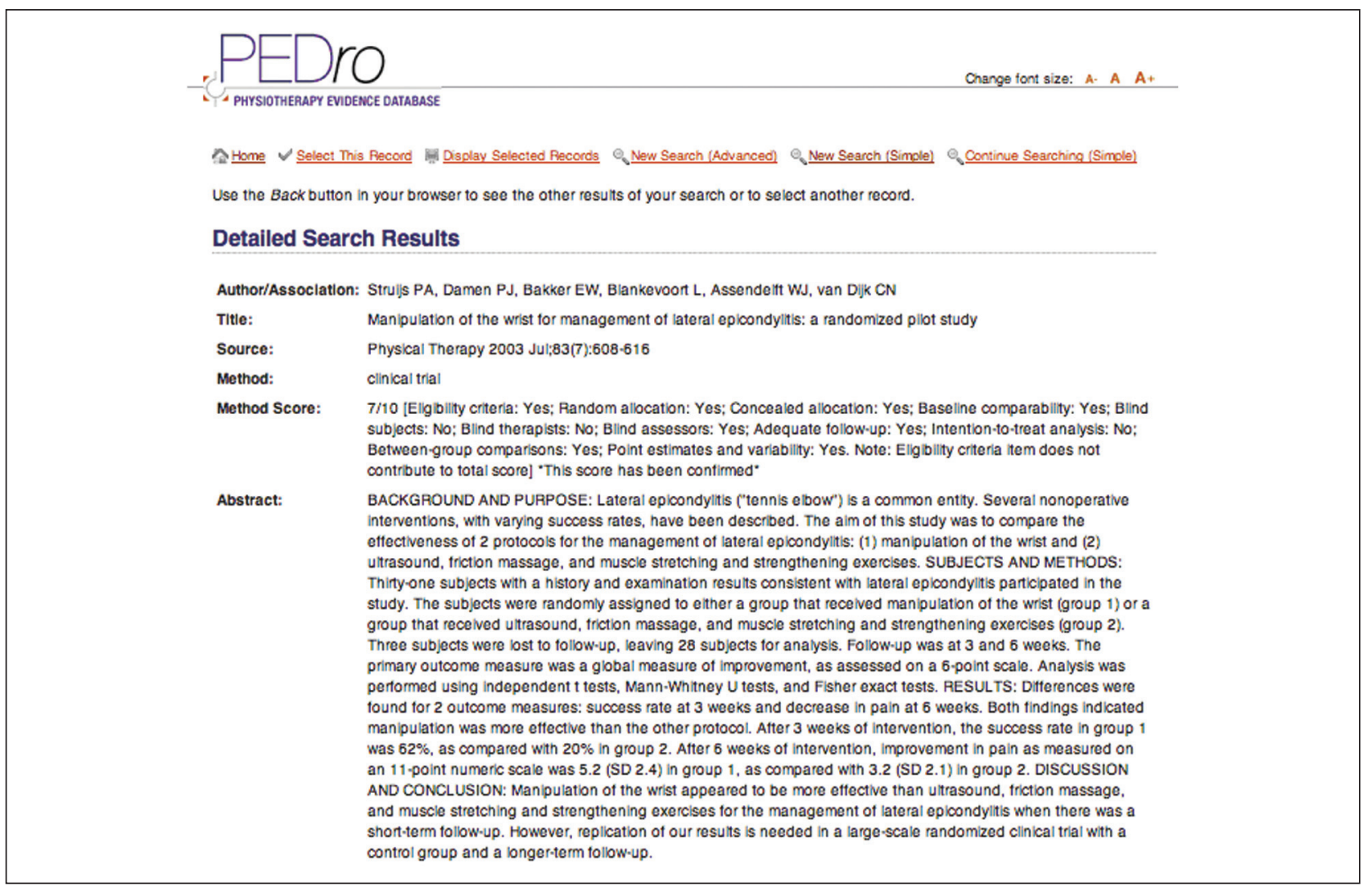

Figura 3 - Detalhes do resultado da busca 


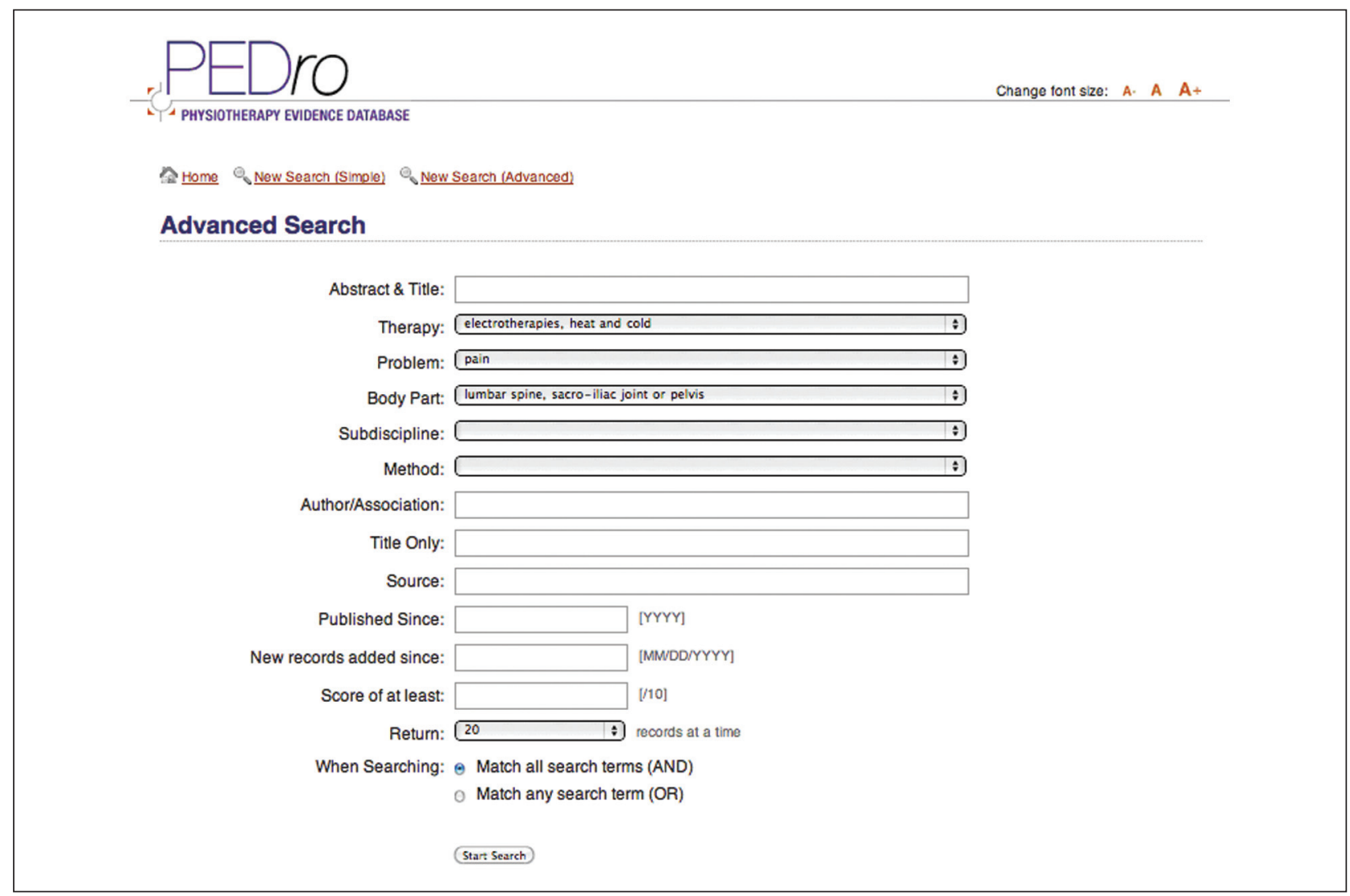

Figura 4 - Apresentação do resultado da busca

disponíveis para busca são: "abstract \& title" (resumo e título), "therapy" (terapia), "problem" (problema), "body part" (parte do corpo), "subdiscipline" (área da fisioterapia: ortopedia, neurologia, ginecologia, ergonomia e saúde ocupacional, entre outras), "method" (tipo de estudo), "author/association" (autor ou associação), "title only" (somente no título), "source" (fonte), "published since" (publicado desde), "new recorded added since" (novos registros adicionados desde), "score of at least" (pontuação mínima), "return" (número de artigos encontrados por página) e "when searching - match all search terms" (AND) (procurar utilizando todos os termos) "or match any search terms (OR)" (procurar por qualquer termo). As páginas de resultados são dispostas da mesma forma apresentada no exemplo anterior (Figura 3).

\section{Escolhas em fisioterapia}

A base de dados Physiotherapy Choices (Escolhas em Fisioterapia <http://www.physiotherapychoices. org.au>) é uma versão da PEDro criada para o uso dos consumidores dos serviços de fisioterapia, entre eles: pacientes, administradores de serviços de saúde e seguradoras. Com linguagem simples e sem termos técnicos, são fornecidas as melhores evidências científicas para a eficácia das intervenções fisioterapêuticas. 0 Brasil é líder no número de acessos dessa versão para consumidores da PEDro, apesar de o website estar disponível somente em inglês.

\section{Website disponível em diversos idiomas}

Com o objetivo de facilitar o acesso à base e minimizar a barreira do idioma, algumas partes do website da base de dados PEDro estão disponíveis em outros idiomas: mandarim (http://www.pedro. org.au/chinese/), francês (http://www.pedro.org. $\mathrm{au} /$ french/), português (http://www.pedro.org.au/ portuguese/) e em alemão (http://www.pedro.org. $\mathrm{au} /$ german/), porém, para realizar a busca independente do idioma deve-se utilizar os termos de busca em inglês, sendo os resultados também apresentados em inglês. 


\section{Conclusão}

A prática da fisioterapia baseada em evidências é fundamental para a valorização e o crescimento da profissão, melhorando a qualidade dos atendimentos e a satisfação do paciente, reduzindo os custos com o tratamento. Ser um profissional diferenciado no mercado significa realizar condutas eficazes para seus pacientes e, para isso, ter acesso à melhor evidência científica é extremamente necessário.

Com o número crescente de estudos na área da fisioterapia, a dificuldade em encontrar a melhor evidência é uma barreira a ser minimizada com o uso da base de dados PEDro, que é a mais completa em fisioterapia. A forma como os resultados das buscas são mostrados pelo website proporciona um rápido acesso às respostas das perguntas clínicas feitas pelos fisioterapeutas usuários do sistema. A PEDro também facilita o acesso à informação sobre a qualidade dos estudos aos pacientes e outros consumidores dos serviços de fisioterapia por meio de um website especializado (Physiotherapy Choices), que apresenta uma linguagem de fácil entendimento.

\section{Referências}

1. Costa LO, Moseley AM, Sherrington C, Maher CG, Herbert RD, Elkins MR. Core journals that publish clinical trials of physical therapy interventions. Phys Ther. 2010;90(11):1631-40.

2. Herbert R, Jamtvedt G, Mead J, Hagen KB. Practical evidence-based physiotherapyed. Edinburgh: Elsevier Butterworth Heinemann; 2005.

3. Colebrook D. Irradiation and health: an ultra-violet irradiation of school children. Medical Research Council Special Report Series. 1929;131:1-47.

4. Kolind-Sorensen V. Lesions of the lateral ligament of the ankle joint. Ugeskr Laeger. 1975;137(29):1637-8.

5. Spitzer W. Scientific approach to the assessment and management of activity-related spinal disorders. Spine. 1987;12(7 Suppl):S1-59.

6. Maher CG, Moseley AM, Sherrington C, Elkins MR, Herbert RD. A description of the trials, reviews, and practice guidelines indexed in the PEDro database. Phys Ther. 2008;88(9):1068-77.
7. Centro de Fisioterapia Baseada em Evidências (CEBP). [acesso em 30 jan. 2011]. Disponível em: http://www. pedro.org.au/portuguese/about-us/cebp/

8. Michaleff ZA, Costa LOP, Moseley AM, Maher CG, Elkins MR, Herbert RD, et al. CENTRAL, PEDro, PubMed, and EMBASE are the most comprehensive databases indexing randomized controlled trials of physical therapy interventions. Phys Ther. 2011;91(2):190-7.

9. Moseley AM, Sherrington C, Elkins MR, Herbert RD, Maher CG. Indexing of randomised controlled trials of physiotherapy interventions: a comparison of AMED, CENTRAL, CINAHL, EMBASE, Hooked on Evidence, PEDro, PsycINFO and PubMed. Physiotherapy. 2009; 95(3):151-6.

10. Sherrington C, Moseley AM, Herbert RD, Elkins MR, Maher CG. Ten years of evidence to guide physiotherapy interventions: Physiotherapy Evidence Database (PEDro). Br J Sports Med. 2010;44(12):836-7.

11. Verhagen AP, de Vet HC, de Bie RA, Kessels AG, Boers M, Bouter LM, et al. The Delphi list: a criteria list for quality assessment of randomized clinical trials for conducting systematic reviews developed by Delphi consensus. J Clin Epidemiol. 1998;51(12):1235-41.

12. de Morton NA. The PEDro scale is a valid measure of the methodological quality of clinical trials: a demographic study. Aust J Physiother. 2009;55(2):129-33.

13. Macedo LG, Elkins MR, Maher CG, Moseley AM, Herbert RD, Sherrington C. There was evidence of convergent and construct validity of Physiotherapy Evidence Database quality scale for physiotherapy trials. J Clin Epidemiol. 2010;63(8):920-5.

14. Shiwa SR, Costa LO. Reprodutibilidade da Escala de Qualidade PEDro. Dissertação [dissertação]. São Paulo: Universidade Cidade de Sao Paulo; 2011.

15. Sherrington C, Herbert RD, Maher CG, Moseley AM. PEDro. A database of randomized trials and systematic reviews in physiotherapy. Man Ther. 2000;5(4):223-6.

Recebido: 03/01/2011 Received: 01/03/2011

Aprovado: 10/05/2011 Approved: 05/10/2011 